\title{
Fault Diagnosis in Multirate Sampled Data Systems
}

\author{
N. Viswanadham* \\ Department of Computer Science and Automation \\ Indian Institute of Science \\ Bangalore, India 560012 \\ Abstract
}

In this paper we present three new schemes for sensor and actuator fault diagnosis for linear time-invariant systems: multirate parity-space techniques, multirate observers, and multirate detection filters. We show that these approaches offer distinct advantages over conventional fault diagnosis schemes in terms of ease of failure detection, the speed and the class of systems for which detection is possible.

There are two primary contributions in this paper. First, we develop schemes for system-wide fault diagnosis, as compared with single component fault detection and isolation. Secondly, we show that the diagnosis of single faults is a generic system property.

\section{Introduction}

Numerous approaches have been developed to address the problem of detecting and isolating failures in dynamical systems, including the use of fault trees [1], Kalman filters [2], observers $[1,3,4]$, parity space techniques $[5,6]$, and detection filters $[7,8,9,10,11]$. Detailed descriptions of these and other methods can be obtained from the recent books [1, 12] and survey articles $[13,3,2]$.

All failure detection methods exploit redundant data which is obtained either directly, when two or more sensors are available for measurement of a process variable, or analytically, when a process variable is estimated using the mathematical process model. These redundancy relationships may then be exploited to generate residual signals. Under normal operating conditions these residuals are "small" in an appropriate sense and yet display distinct patterns when failures occur. The failure diagnosis process thus consists of two stages ${ }^{\dagger}$.

(i) Residual generation: The residuals are independent of true measurements but reflect the effects of modeling uncertainty, noise, and component failures. In the absence of failures and gross modeling errors the residuals are unbiased showing agreement between measurements and model-based predictions.

(ii) Residual analysis: Due to the effects of noise and model uncertainty the residuals must be carefully examined to determine the presense of failures (detection) and which system components have failed (isolation). See [15] for a discussion of methods used in sclecting detection thresholds.

In this paper we shall concentrate on three fault diagnosis techniques: parity space, observers, and detection filters. While these methods are the most popular and comprehensive, the applicability of both parity-space and detection filter based schemes has been limited in practise by the presence of RHP plant transmission zeros. Moreover the detection of actuator failures is typically much more difficult in the single-rate case. Here we show that both sensor and actuator faults may be detected by observing the behaviour of a residual vector as it evolves in a plane determined by the plant dynamics.

Several recent papers in the literature $[16,17]$ have illustrated that periodically time-varying systems and multirate systems in particular possess an important property: namely, that generically one can select sampling rates such that the resulting system does not possess any transmission zeros. Further work has produced an efficient framework for analyzing such systems via an equivalent single-rate system, which has also led in tum to a parameterization of all compensators stabilizing a given multirate plant $[18,19]$. The presense of RHP zeros is usually manifested in the fault diagnosis problem by restrictions on the rank of certain matrices, constraints which are alleviated via the introduction of multirate sampling.

The paper is organized as follows: following a quick overview of the two bodies of theory to be linked in this report, fault diagnosis and multirate systems, we present the main results of the paper: a discussion of how one might implement the three most popular fault diagnosis schemes, parity space, observers, and detection filters, in the context of multirate sampled data systems.

This work performed while on leave as a Visiting Research Fellow with the Control System Laboratory, GE-Corporate Research and Development Center, Schenectady NY.

Laborawry, GE-Corporate Research and Development Center, Schencendy NY. A third stage, failure recovery or reconfiguration of the system is often included in order to enhance system reliability. We shall not explore this aspect of fault diagnosis in this paper. We refer the prabr to the wor

\author{
K. D. Minto \\ Control Systems Laboratory \\ GE-Corporate Research and Development Center \\ Schenectady, NY 12301 \\ II. Background
}

\section{II.1 Preliminaries}

We assume throughout that we are dealing with a hybrid control system, that is to say, a linear, continous, time-invariant plant connected via appropriate zero-order hold and impulse-sampling operators in a feedback configuration with a digital compensator. Following standard practice we associate the signals present in such systems with sequences of numbers defined at the sampling instants $t=k T, k=0,1,2, \ldots$, where $T$ is the sampling interval or period. We shall therefore refer to a signal $x(t=k T)$ via the notation $x_{k}$ or $x_{k T}$ if the sampling interval is not obvious from the context. This notation is extended to multirate sampled signals by explicitly representing the sampling intervals. In particular, two signals $x(t)$ and $y(t)$ sampled at the intervals $t=k_{1} T_{1}$ and $t=k_{2} T_{2}$ respectively would be represented by the sequences $x_{k_{1}}$ and $y_{k_{2}}$.

We assume that all sampling intervals are rationally related, i.e. $T_{i} / T_{j}$ is a rational number for all $(i, j)$, and further that the sampling operations are synchronized. We shall denote the $z$-transform of a discrete-time signal $x_{k}$ as $\mathcal{X}(z)$ or simply $\mathcal{X}$

The plant is assumed to be represented by a the state-space model

$$
\begin{aligned}
& \dot{x}(t)=A x(t)+B u(t) \\
& y(t)=C x(t)+E u(t)
\end{aligned}
$$

where the state vector $x \in \mathcal{R}^{n}$, the input vector $u \in \mathcal{R}^{p}$, and the output vector $y \in \mathcal{R}^{m}$. It is assumed that the triple $(A, B, C)$ is controllable and observable, and furthermore that the state is observable through each output channel.

\section{II.2 Fault Diagnosis}

The fault diagnosis problem we are addressing is depicted in Figure 1. This system configuration differs from a standard compensator feedback loop in that a dedicated fault-diagnosis unit monitors the plant inputs and outputs. This block is typically comprised of a dynamical system to generate and analyze residual signals.

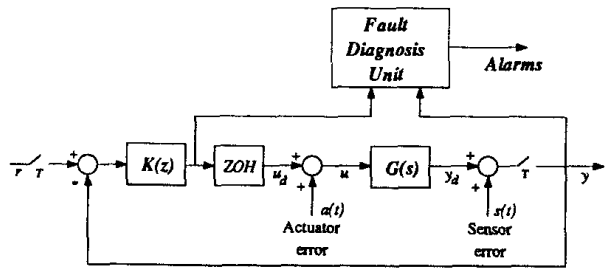

Figure 1 Fault Diagnosis with Implicit Failure Models

In this paper we consider two classes of faults: independent sensor failures, and independent actuator failures. It is possible to model the failure of actuators and sensors as additive signals appearing at appropriate places in the model as indicated in Figure 1 . Let $u_{d}(t)$ be the desired plant input and $u(t)$ be the actual plant input, as modified by the actuator crror signal $a(t)$. By appropriate choice of the elements $a_{i}(t)$, we can represent various failure modes for the $i^{\text {th }}$ actuator. In particular, if the $i^{\text {th }}$ actuator freczes at its nominal position, producing no incremental output, then $a_{i}(t)=-u_{d_{i}}(t)$. If there is a bias $b_{i}$ appearing on the actuator for some reason, then $a_{i}(t)=b_{i}$. Finally, if the $i^{t h}$ actuator is stuck at a constant value $b_{i}$, then $a_{i}(t)=b_{i}-u_{d_{i}}(t)$. Multiple failures can be modeled by specifying several elements of $a(t)$ to be nonzero. Similarly, let $y_{k}$ be the sampled version of the actual sensor output, incorporating the error signal $s(t)$. Sensor failures can be represented in a similar manner by appropriate choice of the signals $s_{i}(t)$. The variables $u_{d}$ and $y$ thus represent the "external" signals, i.e. those that are available for fault diagnosis purposes, and $u$ and $y_{d}$ are "intemal" or unaccessible signals.

Suppose that $G(s)$ is the transfer function matrix of the plant, and that $G(z)$ is it's zero-order hold discrete-time equivalent. Then under normal 
operating conditions we can compute the discretized version of the internal signal $y_{d}$ as

$$
y_{d_{k}}=\mathcal{Z}^{-1}\left\{G(z) \mathcal{U}_{d}\right\}
$$

where $\mathcal{Z}^{-1}(\cdot)$ is the inverse z-transform operator. This computed value may then be compared to the actual measured value and used to form a residual signal referred to as the parity vector, vis.

$$
p_{k}=y_{k}-y_{d_{k}}=y_{k}-\mathcal{z}^{-1}\left\{G \mathcal{U}_{d}\right\}
$$

When the plant transfer function is known exactly, there is no noise, and no failures, then the parity vector $p$ is zero. Under normal conditions however $p$ will be non-zero due to the presence of noise and modelling errors. When a failure occurs however, $p$ will have a relatively large magnitude representing inconsistencies among the actuator inputs and sensor outputs with respect to the unfailed model. Different failures produce parity vectors with decidedly different characteristics, which may then be further used to isolate the failure to a particular system component.

Several researchers have studied the problem of failure isolation based on the direction of propagation of $p(k)$ and also using voting schemes $[20$, $5,6,21]$. However, clean results are available only for systems which are decouplable by an output-injection map, i.e. systems for which a subset of the transmission zeros are stable [1]. We remove this restriction here by allowing periodic output injection maps for stabilization.

An closely related approach to residual generation is based on the use of observers as in Figure 2.

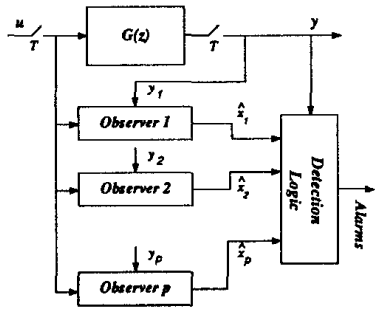

Figure 2 Observer-Based Fault-Diagnosis

In this approach, a bank of observers is used to generate estimates $\hat{x}_{i}$ of the internal states of the plant. These are then used to compute (based on knowledge of the system model) estimates $\hat{y}_{i}$ of the plant output vector and are compared to the actual output $y$ within a detection logic block. We note however that the observer-based scheme as pictured above is useful primarily for sensor fault diagnosis. All the inputs $u$ and only a subset of the outputs $y_{i}$ are used by the $i^{t h}$ observer to generate $\hat{x}_{i}$, the estimate of the state vector $x$. Naturally, the conditions for reconstructability of the state vector from this partial information must be satisfied. Further, one can design single observer based schemes for sensor fault detection and isolation. If one desired to perform combined actuator and sensor fault diagnosis using observer based techniques, one has to appeal to unknowninput observer theory $[22,23]$. Here again the system has to satisfy a variety of conditions involving stability of transmission zeros and the rank of certain system matrices.

The detection filter offers yet another approach for residual generation by comparing the outputs of a linear dynamical model with the actual system measurements, as in Figure 3.

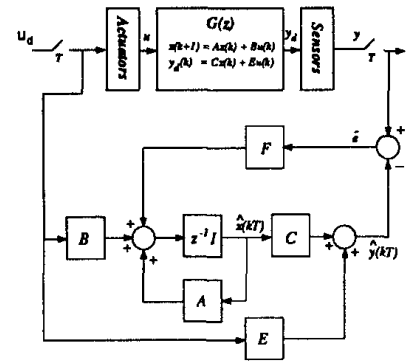

Figure 3 Detection Filter
The detection filter differs from the observer-based approach in that the residuals $\vec{e}$ are fed back to the mode! input through the feedback gain matrix $F$, which is chosen in order that the error $\bar{e}$ propagates along distinct directions for each sensor or actuator failure. In other words the residuals are filtered back through the model in such a way that when one of the components monitored by the detection filter fails, the residual vector is fixed in direction. This approach is also equivalent to the generalized parity vector approach as is shown in [6]. Here also clean isolation methods for sensor/actuator failures are available for systems if a certain subset of transmission zeros are stable. More precisely, most detection filters are designed such that the residuals resulting from actuator failures propagate along fixed directions where as those from sensor failures lie in planes.

\section{II.3 Multirate Sampled Data Systems}

In this paper we shall consider only a special case of a multirate system, as illustrated in Figurc 4. Such a configuration is referred to as a fast-output sampled system, in that all the input signals are assumed to be sampled at the same base time period $T$, whereas the output signals $y_{1}$ through $y_{p}$ are sampled at the rates $T_{/ n_{1}}, \ldots, T_{/ n_{p}}$ respectively, where $n_{1}, \ldots, n_{p}$ are arbitrary non-zero integers.

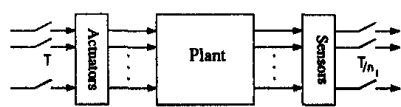

Figure 4 Multirate Sampled Datz System

In case the sampling operations are synchronous and are integer multiples of some base rate $T^{3}$ the underlying system can be viewed as periodically time-varying since the sampling operations are periodic. Thus we can invoke some elegant results from the periodic systems literature to analyze the behaviour of synchronous multirate systems. For purposes of illustration, consider the SISO multirate sampled system $G$ in Figure 5.

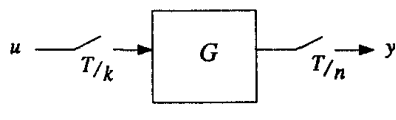

Figure 5 SISO Multirate Sampled System

We now describe an elegant way of constructing a "single-rate" equivalent of $G$. This method is quite popular and has been used by a number of authors, see e.g. $[24,25,17,19,16]$. Let $l_{+}:=\left\{x=\left(x_{0}, x_{1}, \ldots\right)\right.$ : $x ; \in \Re\}$ represent the set of all one-sided sequences. Assuming that $G$ is causal, then $y \in l_{+}$if $u \in l_{+}$. If $n$ denotes the sampling rate increase at the output, define a "lifting" operator $W_{n}$ on the output space as follows

$$
\begin{aligned}
W_{n} & : l_{+} \rightarrow l_{+}^{n}:\left[y_{0}, y_{1}, y_{2}, \ldots\right] \\
& \rightarrow\left[\left(\begin{array}{c}
y_{0} \\
y_{1} \\
\vdots \\
y_{n-1}
\end{array}\right),\left(\begin{array}{c}
y_{n} \\
y_{n+1} \\
\vdots \\
y_{2 n-1}
\end{array}\right), \ldots\right] \\
& =:\left[\mathbf{y}_{\mathbf{o}}, \mathbf{y}_{1}, \ldots\right]
\end{aligned}
$$

Thus we see that the output samples (at intervals $T / n$ ) are rearranged into a vector of $n$ samples at the longer interval $T$. With the input sampled at the intervals $T / k$ a similar operator $W_{k}$ may be defined on the input space. The action of these two operators may be summarized by the following commutative diagram

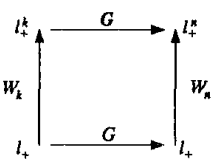

It may readily be shown that the operators $W_{n}$ and $W_{k}$ are invertible so we can define the new operator $\mathrm{G}:=W_{n} G W_{k}^{-1}$ which we shall refer to as the lifted or single-rate equivalent of $G$.

Such a construction might seem to be of rather academic interest, but is actually quite useful, particularly in light of the fact that a state-space 3 The synchronous sampling assumption is fairly standard, yet rarcly achieved in practise, due to
hardware cost constraints. 
realization for the so-called lifted system may be readily obtained from the state-space realization of the underlying linear shift-invariant system $G$. The significance of the relation illustrated in this commutative diagram is that the properties of the multirate system (stability, etc.) may be deduced by examination of the equivalent single-rate system, to which existing analysis tools may readily be applied.

The general formula for the state-space realization of $\mathbf{G}$ is quite complicated, and would not serve to enlighten the reader here. We refer instead to [26] and present only the formula for the fast-output sampled case. Indeed, if $G$ has the state-space realization

$$
G \leftrightarrow\left[\begin{array}{cc}
A & B \\
C & E
\end{array}\right]
$$

then the lifted system $G$ has the state-space realization

$$
G \leftrightarrow\left[\begin{array}{cc}
A^{n} & A^{n-1} B \\
\mathbf{C A} & \mathbf{E} \\
\vdots & \mathbf{C B} \\
\mathbf{C A}^{\mathrm{n}-1} & \mathbf{C A}^{\mathrm{n}-2} \mathrm{~B}
\end{array}\right]
$$

Hence we consider a fault-detection scheme such as illustrated in Figure 6

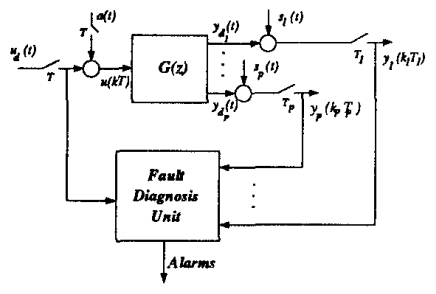

Figure 6 Multirate Fault Diagnosis

In this case we have collapsed the plant, sensors and actuators into a generic system ${ }^{2}$. Recognizing the uniform sampling we represent the input in vector form, whereas the outputs are considered individually due to non-uniform sampling.

\section{Main Results}

\section{III.1 Parity-Space Techniques}

To develop the theory of fault diagnosis using parity-space techniques, we shall first focus on the problem of detecting sensor faults, assuming that all the actuators are functioning properly. Recalling the definition of the parity vector, we seek a means for computing the uncorrupted plant outputs $y_{d_{1}}$ for comparison with the measured outputs $y_{i}$. Suppose that $g_{i}$ represents the transfer function from the inputs to the $i^{\text {th }}$ plant output, with state-space realization given by

$$
g_{i} \leftrightarrow\left[\begin{array}{cc}
A & B \\
c_{i} & e_{i}
\end{array}\right]
$$

Suppose further that $\mu_{i}$ is the observability index for the plant through the $i^{\text {th }}$ output. Then it follows that the matrix

$$
\vartheta_{i}=\left[\begin{array}{c}
c_{i} \\
c_{i} A \\
c_{i} A^{2} \\
\vdots \\
c_{i} A^{\mu_{i}-1}
\end{array}\right]
$$

has full rank, i.e. the rows are linearly independent. However, the row vector $c_{i} A^{\mu_{i}}$ is linearly dependent on the rows of $\vartheta_{i}$. Consequently, there exist scalars $\beta_{i, 0}, \ldots, \beta_{i, \mu_{i}}$ not all zero, such that

$$
\beta_{i, 0} c_{i}+\beta_{i, 1} c_{i} A+\ldots+\beta_{i, \mu_{i}} c_{i} A^{\mu_{i}}=0
$$

1 In fact, such realizations may be defined even when $G$ is a linear shiff-varying operator. See [18] for details.

We hope that the reader will forgive a slight abuse of notation here, in that we have considered the "plant" $G(z)$ to be the discrete-time equivalent of the true system, with input $u(k T)$ discretized at the input sampling period $T$. The outputs are labeled as the continous-time signals $y_{d}(t)$, recognizing

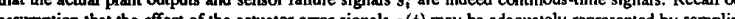
hem at the same rate as the nominal input $t_{\text {d }}$.
Suppose now that we multirate sample the output $y_{i}$ at a rate $\mu_{i}+1$ times the input sampling rate $T$. The state-space realization for the resulting equivalent single-rate system may expressed as

$$
\left[\begin{array}{c}
x((k+1) T) \\
y_{i(k T)} \\
y_{i}\left(k T+\frac{T}{\mu_{i}+1}\right) \\
\vdots \\
y_{i}\left(k T+\frac{\mu_{i} T}{\mu_{i}+1}\right)
\end{array}\right]=\left[\begin{array}{cc}
A^{\mu_{i}+1} & A^{\mu_{i} B} \\
c_{i} & e_{i} \\
c_{i} A & c_{i} B \\
\vdots & \vdots \\
c_{i} A^{\mu_{i}} & c_{i} A^{\mu_{i}-1} B
\end{array}\right]\left[\begin{array}{l}
x(k T) \\
u(k T)
\end{array}\right]
$$

and we can then define a residual

$\rho_{i}\left(k T+\frac{\mu_{i} T}{\mu_{i}+1}\right)=\sum_{j=0}^{\mu_{i}} \beta_{i, j} y_{i}\left(k T+\frac{j T}{\mu_{i}+1}\right)-\left[\beta_{i, 0} e_{i}+\sum_{j=1}^{\mu_{i}} \beta_{i, j} c_{i} A^{j-1} B\right] u(k T)$

This residual will be identically equal to zero if the $i^{\text {th }}$ sensor is good, and non-zero otherwise. Hence, barring computational time, a failure check can be run on any particular sensor after $\mu_{i}$ "fast-samples" of the base sampling interval $k T$ have transpired, where $\mu_{i}$ is the observability index for the plant through that particular channel. The payoff is a much faster indication of sensor faults. Comparing this scheme with a single-rate approach we would require $\mu_{i}$ input samples to collect the same amount of data. Alternately, if we were to just increase the input and output sampling rates by $T / \mu_{i}$, we would get faster fault-diagnosis, at the expense of increased computation. This can be easily seen by noting that the fast sampled version of the plant has $\mu_{i}+2$ block columns and $\rho_{i}$ has components $u(k T), u\left(k T+\frac{T}{\mu_{i}+1}\right), \ldots u\left(k T+\frac{\mu_{i} T}{\mu_{i}+1}\right)$. In this case we must produce control inputs much faster, resulting in much more computation.

The above scheme seems quite appealing intuitively, in that we are effectively oversampling the output signals. Given piecewise-continuous inputs to the system, it follows that the outputs may be predicted after a sufficient time has elapsed to allow determination of the systems initial state. In the absence of unmodeled dynamics, sensor noise or failures then, the value of each output may be readily determined throughout the remainder of the sampling interval. By continuing to sample the system in this period we can detect more quickly any deviation due to a sensor failure.

\section{III.2 System-wide Fault Diagnosis}

In the previous section, we considered the detection of a particular sensor failure from a residual signal $\rho$. From a practical point of view, it is of interest to develop a systematic method for detection and isolation of all the sensor and actuator failures. Here we present such a method using the residuals $\rho_{i}$ defined in (11). We make the assumption here that there are no simultaneous failures present in the system. In other words, there is only one failed component, either a sensor or an actuator, present in the system. Multiple simultaneous component failures are not treated here.

Suppose our system has $p$ outputs and $m$ inputs. Then we have $p$ residuals, $\rho_{i}$, defined by (11). The following logic may be used to detect and isolate failures in this case:

L1 $\rho_{i}=0, i=1,2, \ldots, p$, then all sensors and actuators are good.

L2 $\rho_{j} \neq 0 ; \rho_{i}=0 ; i \neq j, i=1,2, \ldots, p$, then sensor $j$ is bad.

L3 $\rho_{j} \neq 0 ; \forall j$ then one of the actuators is bad.

To isolate actuator failures using the residuals $\rho_{i}$ we need to do a little more work. Suppose there is a failure mode on the $i^{\text {th }}$ actuator. Recalling Figure 1, we have the actuator failure model

$$
u(k T)=u_{d}(k T)+a(k T)
$$

where $a(k T)$ is a column vector with $a_{i}(k T) \neq 0$ and $a_{j}(k T)=0 ; j \neq i$. Substituting (12) in (11) and noting that the right-hand side of (11) with $u(k T)$ replaced by $u_{d}(k T)$ is zero, we get

$$
\begin{gathered}
\rho_{i}\left(k T+\frac{\mu_{i} T}{\mu_{i}+1}\right)=-\left[\beta_{i, o} e_{i}+\sum_{j=1}^{\mu_{i}} \beta_{i, j} c_{i} A^{j-1} B\right] a(k T) ; \\
i=1,2, \ldots, p
\end{gathered}
$$

We can rewrite equation (13) in vector form as

$$
\rho=L a(k T)
$$

where the constant matrix $L$ is defined via

$$
\begin{gathered}
L=\beta \cdot I_{o}, \quad \beta=\operatorname{block} \operatorname{diag}\left(\beta_{1}, \beta_{2}, \ldots, \beta_{p}\right), \\
\beta_{i}=\left[\begin{array}{llllll}
\beta_{i, 0}, \beta_{i, 1}, \ldots, \beta_{i, \mu_{i}}
\end{array}\right] \\
I_{0}=\left[\begin{array}{lllllll}
e_{1}^{\prime} & \left(\vartheta_{1} B\right)^{\prime} & e_{2}^{\prime} & \left(\vartheta_{2} B\right)^{\prime} & \cdots & e_{p}^{\prime} & \left(\vartheta_{p} B\right)^{\prime}
\end{array}\right]^{\prime}
\end{gathered}
$$


Let $L_{i}$ denote the $i^{\text {th }}$ column of $L$. For a failure in the $i^{\text {th }}$ actuator, we have

$$
\rho=L_{i} a_{i}(k T)
$$

Thus we see that the residual vector propagates along $L_{i}$. By finding the direction of propagation of $\rho$, we can isolate the actuator failure. Simply stated, if $\rho$ lies along $L_{i}$, then it signifies a failure in the $i^{\text {th }}$ actuator. To distinguish among various actuator failures however, we need the columns of $L$ to be linearly independent. This requires that the given system $G(s)$ satisfy certain mild conditions. We state the result in the form of a theorem:

Theorem 2.1 Consider a discrete time system $G(z)$ with output dimension $p$ greater than or equal to input dimension $m$. Then a parity vector based failure diagnostic scheme exists for $G(z)$ if and only if $G$ is input observable, i.e. iff

$$
\operatorname{rank}\left[\begin{array}{c}
E \\
C B \\
C A B \\
\vdots \\
C A^{n-1} B
\end{array}\right]=m
$$

It follows from (L1-L3) that the diagnosis of sensor failures needs no technical conditions. The conditions of input observability and $\operatorname{dim}(y)>$ $\operatorname{dim}(u)$ are required to ensure that the columns of $L$ are linearly independent. The theorem proof is then complete with the following lemmas.

Lemma 2.2 G is input observable if and only if $\operatorname{rank}\left(I_{o}\right)=m$.

Proof: This can be easily seen by noting that $I_{0}$ can be obtained from the input-observability matrix (17) by performing elementary row operations and using (9) and eliminating the zero rows. Thus $I_{o}$ is row-equivalent to the input-observability matrix.

Lemma 2.3 Rank $(L)=m$ is a generic property if and only if (i) $G$ is input-observable, and (ii) $p \geq m$.

Proof: Suppose that $G$ is not input-observable, i.e. that (17) does not hold. Then by Lemma 2.2 the rank of $I_{0}<m$. Also from the structure of $\beta$ we have $\operatorname{rank}(\beta)=p$. Utilizing standard results on rank for matrix products we have whenever $p \geq m$

$$
\begin{aligned}
\operatorname{rank}(L) & \leq \min \left(\operatorname{rank}\left(I_{o}\right), \operatorname{rank}(\beta)\right) \\
& \leq \min \left(\operatorname{rank}\left(I_{o}\right), p\right) \leq \operatorname{rank}\left(I_{o}\right) \\
& <m
\end{aligned}
$$

Also, if $p<m$, then $\operatorname{rank}(L)$ is always $<m$. Thus we have established the necessity.

To prove sufficiency, suppose $p>m$ and (17) holds. Consider any arbitrary order $m$ minor of $L$ formed by extracting rows $i_{1}, i_{2}, \ldots, i_{m}$, which we denote by $L_{(1, \ldots m)}^{\left(i_{1} \ldots i_{m}\right)}$. We show that this minor is non-zero and is an algebraic variety in the elements of $A, B, C$. Thus the generic rank of $L$ is $m$.

To proceed, note by the Binet-Cauchy formula that

$$
L_{(1 \cdots m)}^{\left(i_{1} \cdots i_{m}\right)}=\sum_{1 \leq \cdots \leq n} \beta_{\left(j_{1} \cdots j_{m}\right)}^{\left(i_{1} \cdots i_{m}\right)} I_{0(1 \cdots m)}^{\left(j_{1} \cdots j_{m}\right)}
$$

where $\bar{n}=\sum_{i} \mu_{i}+p$ is the row dimension of $L$. To show that this minor is not identically zero, we note that for each $i$, there exists at least one $\beta_{i j} \neq 0$. Suppose this non-zero term is $\beta_{i_{k} j_{k}}$. Then

$$
\beta_{\left(j_{1} j_{2} \cdots j_{m}\right)}^{\left(i_{1} i_{2} \ldots i_{m}\right)}=\prod_{k=1}^{m} \beta_{i_{k} j_{k}} \neq 0
$$

The corresponding minor of $I_{0}$ is zero only on a hypersurface in the parametric space of $A, B, C, E$. Thus $L_{(12}^{\left(i_{1} i_{2} \ldots i_{m}\right)} \not \equiv$. Further, from (19) it follows that all such minors of $L$ are zero only on a hypersurface, and hence the lemma is established.
We have thus shown that system-wide fault diagnosis of sensors and actuators is possible using multirate parity relations, i.e equation (11). We point out that the simplicity of the technique is a consequence of sampling the output faster than the input. In other words, corresponding results for the single rate sampled data system would be difficult to derive. Since input-observability is a generic system property, i.e it holds almost always for any given physical system, we can state the following theorem which follows directly from the preceding development.

Theorem 2.4 A multirate parity-space fault diagnosis scheme generically exists for any system that has at least as many outputs as inputs.

Thus we see that for tall ${ }^{\ddagger}$ systems single sensor/actuator fault diagnosis is a generic property. We believe this to be a new result, and is certainly encouraging for those engaged in the control system design process for life critical applications such nuclear reactors, aircraft and spacecraft.

We are aware that any model-based diagnostic scheme such as this one has to deal with the issue of robustness $[10,11,4]$. We are currently looking into this issue in the context of multirate systems. Further, from the point of view of distinguishing between faults, the column vectors $L_{i}$ should be "separated" as much as possible, or more precisely, they should be as nearly orthogonal as possible.

\section{III.3 Observers}

In this case we construct an estimate $\hat{x}(k T)$ for the system state, and estimate the outputs directly for monitoring purposes. There are two distinct cases to consider.

Case 1: Suppose that $\left(c_{i}, A\right)$ is observable for some output $i$. Choose $\mu_{i}=n$, where $n$ is the order of the system, and define the matrices

$$
\bar{C}_{i}:=\left[\begin{array}{c}
c_{i} \\
c_{i} A \\
\vdots \\
c_{i} A^{n-1}
\end{array}\right], \quad \bar{E}_{i}:=\left[\begin{array}{c}
e_{i} \\
c_{i} B \\
\vdots \\
c_{i} A^{n-2} B
\end{array}\right]
$$

Then $\bar{C}_{i}$ is non-singular, and we can form the state-estimate directly, given the output measurement vector

$$
Y_{i}(k T):=\left[\begin{array}{c}
y_{i}(k T) \\
y_{i}\left(k T+\frac{T}{\mu_{i}+1}\right) \\
\vdots \\
y_{i}\left(k T+\frac{\mu_{i} T}{\mu_{i}+1}\right)
\end{array}\right]
$$

and the input vector $u(k T)$. The state estimate may be obtained directly from the relation

$$
\widehat{x}(k T)=\bar{C}_{i}^{-1}\left[Y_{i}(k T)-\bar{E}_{i} u(k T)\right]
$$

Given the state estimate, we can then compute the output estimate and error residual as shown below.

$$
\begin{aligned}
& \widehat{y}_{j}(k T)=c_{j} \widehat{x}(k T), \quad i \neq j, j=1,2, \ldots, p \\
& \rho_{j}(k T)=y_{j}(k T)-\widehat{y}_{j}(k T), i \neq j
\end{aligned}
$$

It is interesting to note that such an approach is not used in general for discrete-time systems, because the state-estimate $\hat{x}(k T)$ must first be explicitly formed as the output of a separate dynamical system since the system outputs may only contain partial state information (equivalently, the $C$ matrix does not have full-column rank). Here we form the state estimate directly, since we can ensure through proper choice of the output sampling rate $T / \mu_{i}$ that the multirate-sampled outputs contain full-state information. Indeed, save for registers to store the $\mu_{i}$ samples that comprise the composite output vector $Y_{i}$, the computation of the state-estimate is purely algebraic.

Case 2: We can get estimates faster by duplicating this approach, in effect having a bank of $p$ observers operating concurrently, one for each

\footnotetext{
Those with at least as many outputs as inputs. This is the most cornmon case in practice.
} 
output $y_{i}$. Let $C^{1}$ be the matrix $C$ with the first row $c_{1}$ removed. Supposing that $\left(C^{1}, A\right)$ is observable, there exist integers $\delta_{2}, \ldots, \delta_{p}$ such that

$$
\text { rank }\left[\begin{array}{c}
c_{2} \\
c_{2} A \\
\vdots \\
c_{2} A^{\delta_{2}-1} \\
c_{3} \\
\vdots \\
c_{p} A^{\delta_{p}-1}
\end{array}\right]=n .
$$

Let

$$
Y^{1}=\left[\begin{array}{c}
Y_{2} \\
Y_{3} \\
\vdots \\
Y_{p}
\end{array}\right] ; \bar{C}^{1}=\left[\begin{array}{c}
\bar{C}_{2} \\
\bar{C}_{3} \\
\vdots \\
\bar{C}_{p}
\end{array}\right] ; \bar{E}^{1}=\left[\begin{array}{c}
\bar{E}_{2} \\
\bar{E}_{3} \\
\vdots \\
\bar{E}_{p}
\end{array}\right]
$$

and hence

$$
Y^{1}=\bar{C}^{1} x(k T)+\bar{E}^{\perp} u(k T)
$$

which we note has the same form as in (10). Hence we can compute the state estimate $\widehat{x}(k T)$ as in the previous section,

$$
\widehat{x}(k T)=\left(\bar{C}^{1}\right)^{-1}\left[Y^{1}-\bar{E}^{1} u(k T)\right]
$$

Since the vector $Y^{1}$ contains information up to $\delta=\max \left(\delta_{i}\right)$, where $\delta<\mu_{i}$, it follows that the state-estimate can be computed faster, after only $\delta$ of the fast output samples in this case.

A particularly attractive approach would be to use a $\mathrm{TMR}^{\S}$ sensor for one output channel to obtain one valid output, and use that signal to estimate the others for fault diagnosis. We summarize these results in the following theorem.

Theorem 3.1 Consider an observable system $G(z)$. Then there always exists a (multirate) observer based diagnosis scheme for sensor fault detection and isolation.

The question arises as to whether system-wide actuator fault diagnosis is possible using observer schemes. For actuator diagnosis to be possible, we need to extend unknown-input observer theory $[22,23]$ to the multirate sampling framework. Research is continuing on this aspect of the problem.

\section{III.4 Detection Filter}

The multirate detection filter is formed in a natural manner by multirate sampling of the system outputs, with the structure as illustrated by Figure 7

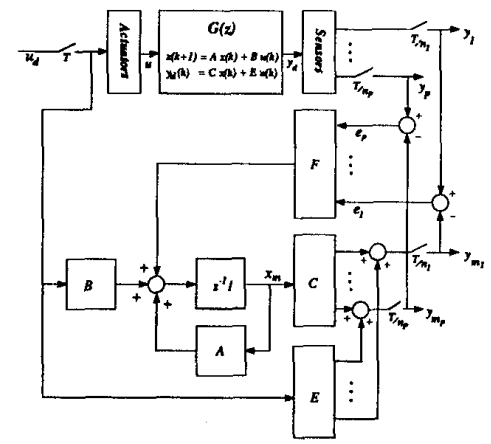

Figure 7 Multirate Detection Filter

Consider for a moment the derivation of the single-rate detection filter. Suppose that the plant $G$ has the standard state-space realization" as in Figure 7. Then the detection filter may be described by the equations

$$
\begin{aligned}
x_{m}(k+1) & =(A-F C) x_{m}(k)+(B+F E) u_{d}(k)+F y(k) \\
y_{m}(k) & =C x_{m}(k)+E u_{d}(k) \\
e(k+1) & =\left(x-x_{m}\right)(k+1) \\
& =(A-F C) e(k)+(B+F E)\left(u-u_{d}\right)(k)
\end{aligned}
$$
Triple-Modular Redondant
Note that this is somewhat non-standard, in that we have a throughput term $E$ present. Previous
derivations of the detection fller considered only the case of stricly-proper plants. The non-zero
throughput term will be significant here however, in that it arises naturally when considering the throughput term will be significant here ho
single-rate equivalent of the multirate system.
Combining the plant dynamics and the detection filter equations above we can define the error signal dynamics $e$ as follows:

$$
\begin{aligned}
e(k+1) & =\left(x-x_{m}\right)(k+1) \\
& =(A-F C) e(k)+(B+F E)\left(u-u_{d}\right)(k) \\
\bar{e}(k) & =C\left(x-x_{m}\right)(k)+E\left(u-u_{d}\right)(k) \\
& =C e(k)+E\left(u-u_{d}\right)(k)
\end{aligned}
$$

If $u=u_{d}$, then we have

$$
\begin{aligned}
e(k+1) & =(A-F C) e(k) \\
\bar{e}(k) & =C e(k)
\end{aligned}
$$

Given such a structure, the feedback matrix $F$ may be chosen to stabilize the error dynamics, i.e. such that $(A-F C)$ is stable, and moreover such that the error vector $\bar{e}$ exhibits distinct characteristics for each sensor or actuator failure. For example, if $F$ is chosen such that (i) $(A-F C)$ is stable and (ii) the $p \times m$ transfer matrix

$$
H(z)=C(z I-A+F C)^{-1}(B+F E)+E
$$

has a decoupled structure, then the actuator failures manifest themselves along the unit vectors in the $m$-dimensional plane. It may also be shown that $F$ may be chosen so that the error vector possesses similar properties for sensor failures as well $[7,8,9]$.

Now, let us see how the detection filter could be designed using multirate sampling. With reference to Figure 7 , choose $n_{i}=\delta_{i}, i=$ $1, \ldots, p$ where the $\delta_{i}$ 's are the system observability indices, and define $n=\operatorname{lcm}\left(n_{1}, \ldots, n_{p}\right)$. Then the single-rate equivalent $\mathbf{G}$ of the multirate plant $G$ has the state-space realization

$$
\mathbf{G} \leftrightarrow\left[\begin{array}{cc}
\mathbf{A}^{\mathbf{n}} & \mathbf{A}^{\mathbf{n}-1} \mathbf{B} \\
\mathbf{C}_{1} & \mathbf{E}_{1} \\
\mathbf{C}_{1} \mathbf{A} & \mathbf{C}_{1} \mathbf{B} \\
\vdots & \vdots \\
\mathbf{C}_{1} \mathbf{A}^{\delta_{1}} & \mathbf{C}_{1} \mathbf{A}^{\delta_{1}-1} \mathbf{B} \\
\mathbf{C}_{2} & \mathbf{E}_{2} \\
\vdots & \vdots \\
\mathbf{C}_{\mathrm{p}} \mathbf{A}^{\delta_{\mathrm{p}}} & \mathbf{C}_{\mathrm{p}} \mathbf{A}^{\delta_{\mathrm{r}}-1} \mathbf{B}
\end{array}\right]=:\left[\begin{array}{cc}
\bar{A} & \bar{B} \\
\bar{C} & \bar{E}
\end{array}\right]
$$

Hence the equation governing the error dynamics becomes $\bar{A}-\bar{F} \bar{C}$. Given this choice of sampling rates we can ensure that $\bar{C}$, the observability matrix, is nonsingular. Hence finding a matrix $\bar{F}$ to stabilize the pair $(\bar{A}, \bar{C})$ is trivial. In particular, we could choose $\bar{F}=\bar{C}^{-1} \bar{A}$, which produces deadbeat error dynamics.

In case $u=u_{d}$ (no actuator failures), suppose that the $i^{\text {th }}$ sensor fails, such that $y_{i}(k)=y_{d_{i}}(k)+s_{i}(k)$, where $s_{i}(k)$ is an arbitrary nonzero signal. Assuming that $\bar{F}$ has been chosen as above, then the error dynamics are governed by the equation

$$
\begin{aligned}
e(k+1) & =f_{i} s_{i}(k) \\
\bar{e}(k) & =\overrightarrow{\mathbf{e}}_{i} s_{i}(k)+\bar{C} e(k)
\end{aligned}
$$

where $f_{i}$ is the $i^{\text {th }}$ column of $\bar{F}$, and $\overrightarrow{\mathbf{e}}_{i}$ is the $i^{\text {th }}$ unit vector in the $n$-dimensional space. Further simplification of equation (34) produces

$$
\begin{aligned}
\bar{e}(k) & =\overrightarrow{\mathbf{e}}_{i} s_{i}(k)+\bar{C} f_{i} s_{i}(k-1) \\
& =\overrightarrow{\mathbf{e}}_{i} s_{i}(k)+\bar{A}_{i} s_{i}(k-1)
\end{aligned}
$$

which illustrates that the error vector lies in a plane. If the sensor failures are of the bias type then $s_{i}(k)=s_{i}(k-1)=s_{i}$ for all $k$, and we have

$$
\bar{e}(k)=\left(\overrightarrow{\mathbf{e}}_{i}+\bar{A}_{i}\right) s_{i}
$$

Thus the first $p$ columns of the matrix $(I+\bar{A})$ are the directions along which the error propagates in case of sensor failures. Furthermore, $(I+\vec{A})$ also provides the directions along which the detection filter error settles once the error in the sensors settles down to a steady state value.

In the case of a failure in the $j^{\text {th }}$ actuator, $u(k)-u_{d}(k)=a_{j}(k)$ where $a_{j}(k)$ is an arbitrary signal. The error dynamics in this case are govemed by the equation

$$
\begin{aligned}
e(k+1) & =(\bar{B}+\bar{F} \tilde{E})_{j} a_{j}(k) \\
\bar{e}(k) & =\bar{C}(\bar{B}+\bar{F} \bar{E})_{j} a_{j}(k-1)+\bar{E}_{j} a_{j}(k)
\end{aligned}
$$


the subscripts on the matrices denote the $j^{\text {th }}$ column. This equation illustrates that actuators faults also produce error signals that propagate in a plane. In case of a step-change error in an actuator, we have $a_{j}(k)=a_{j}(k-1)=a_{j}$, a constant. Then (37) simplifies to

$$
\bar{e}(k)=\left(\bar{C} \bar{B}_{j}+(I+\bar{A}) \bar{E}_{j}\right) a_{j}
$$

All step-type actuator failures produce detection filter error along the first $m$ columns of the matrix $(\bar{C} \bar{B}+(I+\bar{A}) \bar{E})$. As in the case of sensor failures, the first $q$ columns of this matrix also determine the directions along which the steady-state detection filter error propagates for any actuator failure that eventually settles down to a constant value. As before, we summarize these results in the form of a theorem.

Theorem 4.1 Given an observable system $G(z)$, there exists a deadbeat detection filter and a choice of input and output sampling rates such that system-wide sensor/actuator fault diagnosis can be made using fixed direction or planar signatures.

It is easy to see the benefit of multirate sampling from equations (35-38). We need only the observability of the system (or equivalently, the nonsingularity of $\bar{C}$ ) as a condition for the existence of the detection filter. The implementation of the failure detection and isolation schemes is then similar to the single-rate case. However, in the single-rate case it is also required that the system satisfy certain decouplability conditions [9].

The failure signatures produced by the multirate detection filter (MRDF) are amenable to simple diagnostic techniques. As with any type of detection system the appearance of a residual with a magnitude greater than some threshold signals failure. In our case, the detection filter generates distinct failure signatures for both actuators and sensors. In the particular case of sensor bias failures these signatures are unidirectional. Isolation in this case is relatively easy; one can use standard vector geometry to determine which of the failure signature vectors is most closely aligned with the residual vector. Planar (actuator) signatures are dealt with in a similar manner.

\section{Conclusions}

We have introduced three new techniques for the detection of failures in linear time invariant systems, based on the use of multirate sampling in the context of traditional observer, parity-space, and detection filter systems. It has been shown that the use of multirate sampling in each of these systems provides a more elegant failure detection system and alleviates the problems inherent with application to systems containing non-minimum phase zeros. In particular, we have shown that the existence of certain types of fault diagnosis schemes is a generic system property.

There are certainly many areas for fruitful research yet to be pursued in the context of multirate fault diagnosis. We are currently focusing on implementation issues via application of the techniques to variable cycle turbine engines. There is (at least conceptually) considerable design freedom here, particularly in the choice of sampling rates, that may be used for instance in obtaining better numerical conditioning of several matrices for which inverses are required. As mentioned previously, there is a need to extend these results to deal with the issues of robustness, and unknowninput observer theory for system-wide actuator fault diagnosis.

We have also considered for the sake of simplicity only a rather simple case of multirate sampling, limited as it were to fast sampling of the system outputs. We present the conjecture that a more general sampling scheme involving multirate sampling of the system inputs and outputs might present further enhancements over single-rate fault diagnosis schemes.

\section{Acknowledgements}

The first author would like to thank Tim Johnson for arranging a Visiting Research Fellowship with the Control Systems Laboratory, GE CR\&D. We are also indebted to Tim for his encouragement and support of this work, and for many fruitful discussions.

\section{References}

[1] N. Viswanadham, V. V. S. Sarma, and M. G. Singh, Reliability of Computer and Control Systems. Amsterdam: North-Holland, 1987.

[2] A. S. Willsky, "A survey of design methods for failure detection in dynamic systems," Automatica, vol. 12, no. 6, pp. 601-611, 1976.

[3] W. Merrill, B. Lehtinen, and J. Zeller, "The role of modem control theory in the design of control for aircraft turbine engines," AIAA Journal of Guidance and Control, vol. 7, no. 6, pp. 652-661, 1984.
[4] N. Viswanadham and K. D. Minto, "Robust observer design with application to fault detection," in Proceedings of American Control Conference, (Atlanta), pp. 1393-1399, 1988.

[5] E. Y. Chow and A. S. Willsky, "Analytical redundancy and the design of robust failure detection systems," IEEE Trans. Automat. Contr., vol. AC-29, no. 7, pp. 603-614, 1984 .

[6] N. Viswanadham, J. H. Taylor, and E. C. Luce, "A frequency-domain approach to failure detection and isolation with application to ge21 turbine engine control systems," Control-Theory and Advanced Technology, vol. 3, no. 1, pp. 45-72, 1987.

[7] R. V. Beard, "Failure accommodation in linear systems through self-organization," Tech. Rep. MVT-71-1, Man Vehicle Laboratory, M.I.T., Cambridge, MA, 1971.

[8] H. L. Jones, Failure Detection in Linear Systems. PhD thesis, M.I.T., Cambridge, September 1973. Department of Aeronautics and Astronautics.

[9] J. S. Meserole, Detection Filters for Fault-Tolerant Control of Turbofan Engines. PhD thesis, M.I.T., Cambridge, June 1981. Department of Aeronautics and Astronautics.

[10] X.-C. Lou, A. S. Willsky, and G. C. Verghese, "Failure detection with uncertain models," in Proceedings of 1983 American Control Conference, (San Francisco, CA), pp. 956-959, June 1983.

[11] X.-C. Lou, A. S. Willsky, and G. C. Verghese, "Optimally robust redundancy relations for failure detection in uncertain systems," Automatica, vol. 22, no. 3, pp. 333-344, 1986.

[12] R. Patton, P. Frank, and R. Clark, Fault Diagnosis in Dynamic Systems: Theory and Application. New York: Prentice Hall, 1989.

[13] R. Isermann, "Process fault detection based on modelling and estimation-a survey," Automatica, vol. 20, no. 4, pp. 387-404, 1984.

[14] C. N. Nett, C. A. Jacobson, and A. Miller, "An integrated approach to controls and diagnostics: The 4-parameter controller," in 1988 American Control Conference, pp. 824-835, June 1988.

[15] A. Emami-Naeini, M. M. Akhter, and S. M. Rock, "Effect of model uncertainty on failure detection: The threshold selector," IEEE Trans. Automat. Contr., vol. AC-33, pp. 1106-1115, December 1988.

[16] B. A. Francis and T. T. Georgiou, "Stability theory for linear time-invariant plants, with periodic digital controllers," IEEE Trans. Automat. Contr., vol. AC-33, pp. 820-832, September 1988.

[17] P. P. Khargonekar, K. Poola, and A. Tannenbaum, "Robust control of linear time-invariant plants using periodic compensation," IEEE Trans. Automat. Contr., vol. AC-30, pp. 1088-1096, November 1985.

[18] R. Ravi, P. P. Khargonekar, K. D. Minto, and C. N. Nett, "Controller parameterization for time-varying multirate plants." Submitted to IEEE Trans. on Auto. Control, 1989.

[19] D. G. Meyer, "Shift-invariant equivalents for a new class of shiftvarying operators with applications to multirate digital control," in Proceedings of 27th Conference on Decision and Control, (Austin, TX), pp. 1697-1701, 1988.

[20] M. Massoumnia, "A geometric approach to the synthesis of failure detection filters," IEEE Trans. Automat. Contr., vol. AC-31, pp. 839846, Sept. 1986.

[21] N. Viswanadham, Reliability of Control Systems. To appear in Supplement 1, Systems and Control Encyclopedia, Pergamon Press, 1990.

[22] N. Viswanadham and R. Srichandar, "Fault-detection using unknowninput observers," Control-Theory and Advanced Technology, vol. 3, no. 2, pp. 91-101, 1987.

[23] P. M. Frank and J. Wunnenberg, "Robust fauit diagnosis using unknown input observer schemes," in Fault Diagnosis in Dynamic Systems - Theory and Application (R. Patton, P. M. Frank, and R. N. Clark, eds.), Prentice-Hall, 1989

[24] G. M. Kranc, "Input-output analysis of multirate feedback systems," IRE Transactions on Automatic Control, vol. AC-3, pp. 21-28, November 1957.

[25] R. A. Meyer and C. S. Burris, "A unified analysis of multirate and periodically time-varying digital filters," IEEE Transactions on Circuits and Systems, vol. CAS-22, pp. 162-168, March 1975.

[26] P. M. Thompson, "Gain and phase margins of multirate sampled-data feedback systems," Int. J. Contr., vol. 44, pp. 833-846, September 1986 